hep-th/0002175, IAS-HEP-00/07, PUPT-1914

February 21, 2000

\title{
Pinned Branes and New Non Lorentz Invariant Theories
}

\author{
Shoibal Chakravarty*, Keshav Dasgupta ${ }^{\star}$, Ori J. Ganor* and Govindan Rajesh ${ }^{\star}$ \\ * Department of Physics, Jadwin Hall \\ Princeton University \\ Princeton, NJ 08544, USA \\ shoibalc@princeton.edu,origa@viper.princeton.edu \\ $\diamond$ School of Natural Sciences \\ Institute of Advanced Study \\ Princeton, NJ 08540, USA \\ keshav,rajesh@sns.ias.edu
}

\begin{abstract}
We describe a mechanism for localising branes in ambient space. When a 3-form flux is turned on in a Taub-NUT space, an M5-brane gets an effective potential that pins it to the center of the space. A similar effect occurs for M2-branes and D-branes with appropriate fluxes. In carefully chosen limits of the external parameters, this leads to new theories that are decoupled from gravity and appear to break Lorentz invariance. For example, we predict the existence of a new $5+1 \mathrm{D}$ theory that breaks Lorentz invariance at high-energy and has a low-energy description of $N$ tensor multiplets with $\mathcal{N}=(1,0)$ supersymmetry. We also predict a new type of theory that, similarly to the little-string theory decouples from gravity by a dynamical (rather than kinematical) argument.
\end{abstract}




\section{Introduction}

The way one usually relates field-theories to branes is to take the low-energy limit. Thus, taking $M_{s} \rightarrow \infty$ for $N$ coincident D3-branes leaves the $U(N) \mathcal{N}=4$ SYM degrees of freedom only [1] and taking the $M_{p} \rightarrow \infty$ limit for $N$ coincident M5-branes leaves the $(2,0)$ degrees of freedom [2]. One can obtain field theories with less supersymmetry by placing the branes at singularities [3]- [6] [7]. A larger class of theories is possible if one relaxes the condition of Lorentz invariance. One can realize the Lorentz noninvariant theories by placing the branes in backgrounds that break Lorentz invariance. The most studied example is Yang-Mills theories on noncommutative spaces [9] obtained by an appropriate scaling limit of branes in backgrounds with an NSNS flux [10]-[12].

The purpose of this paper is to study more configurations of branes at backgrounds that break Lorentz invariance and have an interesting low-energy limit.

The construction that we will use is as follows. Consider a smooth 4D Taub-NUT space (in either M-theory or type-II string theories) that at infinity behaves as a circle fibration over the sphere $S^{2}$ with first Chern class $c_{1}=1$. To be concrete, let us take M-theory. The Taub-NUT space is homogeneous in $6+1$ directions. We can turn on a constant 3 -form $C$ flux along the circle at infinity and two of the homogeneous directions, such that the 4-form field-strength $d C$ is zero at infinity. Because the Taub-NUT circle shrinks to a point at the origin the 4-form field strength cannot remain zero throughout the interior of the Taub-NUT space. In the classical approximation, a solution with this particular boundary conditions forces a nonzero field-strength and therefore also affects the metric. The metric is changed in such a way that a brane that is transverse to the Taub-NUT space (i.e. parallel to the $6+1$ directions) would have a lower tension if it is at the center of the space.

By tuning the external parameters (the $C$ flux at infinity and the radius of the Taub-NUT circle at infinity) we can decouple gravity. In fact, we will suggest two possible limits that decouple gravity. In the first limit the flux is small and the low-energy theory appears to be a new kind of a $5+1 \mathrm{D}$ theory with $\mathcal{N}=(1,0)$ supersymmetry that can roughly be described as the $(2,0)$ theory with a massive hypermultiplet. There is no contradiction with chirality of the hypermultiplet in $5+1 \mathrm{D}$ because we believe the theory (and, in particular, the mass term) breaks Lorentz invariance explicitly. In the second limit the flux is kept finite and the 
decoupling argument is of the same nature as the dynamical argument presented in [8].

Similar constructions can be repeated with the M2-brane and D-branes.

The paper is organized as follows. In section (2) we will describe the setting for the constructions and review the geometry of the Taub-NUT space. In section (3) we will study the spectrum of BPS excitations of the pinned branes and the various energy scales involved. We will show that the spectrum includes a particle with a very low mass. In section (4) we will interpret some of the results of section (2) from the low-energy supergravity solutions. In section (5) we will present the limits of the external parameters that decouples gravity. In

section (6) we discuss the low-energy description of the theories and resolve a puzzle about fermions. In section (7) we remove the M5-branes and study the Taub-NUT space with 3 -form flux, $C_{3}$ on its own. We analyze the spectrum of BPS states and show that the large $C_{3}$ limit can be accompanied with a rescaling of coordinates that makes the energies of the low-lying BPS states finite.

\section{The setting}

\subsection{Review of the Taub-NUT geometry}

The metric of a KK-monopole is the Taub-NUT metric:

$$
d s^{2}=R^{2} U\left(d y-A_{i} d x^{i}\right)^{2}+U^{-1}(d \vec{x})^{2}, \quad i=1 \ldots 3, \quad 0 \leq y \leq 2 \pi .
$$

where,

$$
U=\left(1+\frac{R}{|\vec{x}|}\right)^{-1},
$$

and $A_{i}$ is the gauge field of a monopole centered at the origin. This metric has a few properties that we will utilize.

- It is a circle fibration over $\mathbf{R}^{3}$ with the origin excluded.

- The radius of the fiber shrinks to zero as we approach the origin and becomes a constant $R$ as we approach infinity. 
- If we restrict to $|\vec{x}|=r$ with constant $r>0$ the circle fibration is equivalent to the Hopf fibration of $S^{1}$ over $S^{2}$.

- There is a $U(1)$ isometry $y \rightarrow y+\epsilon$. It has one fixed point at the origin.

- The $U(1)$ isometry acts nontrivially on the tangent space to the point at the origin.

\subsection{Turning on a flux at infinity}

Now suppose that we have a theory of gravity coupled to a vector field $A_{\mu}$ and we are looking for a solution to the equations of motion with boundary conditions such that at infinity we have a circle fibration over $S^{2}$ with $c_{1}=1$ and there is a constant Wilson line $\int A_{y} d y=w$ on the circle at infinity. If $w \neq 0$, we cannot have $F_{\mu \nu}=0$ throughout space because this will force the holonomy $\int A_{y} d y$ to be constant contradicting the fact that the circle shrinks to zero at $\vec{x}=0$. Thus, we expect that the solution with the above boundary conditions will have a nonzero field-strength near the center of the Taub-NUT space. We also expect the Taub-NUT metric to change, as a consequence.

Now let us turn to the setting in our case. We take a Taub-NUT space in M-theory or one of the type-II string theories and we turn on a tensor field at infinity. In M-theory we take the Taub-NUT circle direction to be the $7^{\text {th }}$ and let $0 \ldots 6$ be directions perpendicular to the Taub-NUT space. We can then turn on $C_{167}$ at infinity. In a low energy limit, we will see that there exists a solution with this kind of boundary condition (i.e. being a Hopf fibration at infinity and having the constant $C_{167}$ flux). It is also very plausible that for any value of $C_{167}$ there exists a background of M-theory with these boundary conditions. Next, we add M5-branes along directions $0 \ldots 5$ and ask whether there is a limit of $R$ and $C_{167}$ for which we obtain a theory that is decoupled from gravity.

\section{BPS states}

We wish to study the dynamics of $N$ M5-branes at the center of a Taub-NUT space with a 3 -form field that is constant at infinity and has one direction along the Taub-NUT circle. In order to understand the dynamics and the relevant energy scales of the system we will study various BPS states and fluxes in this theory. 


\subsection{Embedding in M-theory on $T^{7}$}

We would like to use the formula for masses of BPS particles in M-theory on $T^{7}$ (see [13, 14]). We will start with all fluxes turned off and take $T^{7}$ in the form of a product of circles of radii $R_{1} \ldots R_{7}$. We will denote by $M_{p}$ the 11-dimensional Planck scale. We take the Taub-NUT circle to be $R_{7}$. We let the Taub-NUT wrap directions $1 \ldots 6$. Its mass is:

$$
M_{T N}=M_{p}^{9} V R_{7}, \quad V \equiv R_{1} \ldots R_{7} .
$$

The mass of the M5-brane is:

$$
M_{M 5}=M_{p}^{6} R_{1} R_{2} R_{3} R_{4} R_{5}=M_{p}^{6} V R_{6}^{-1} R_{7}^{-1} .
$$

There are $56 U(1)$ charges in the low-energy description of M-theory on $T^{7}$. We will denote them by:

$$
Q^{i}, \widetilde{Q}_{i}, Q_{i j}, \widetilde{Q}^{i j}
$$

They correspond to BPS particles with masses:

$$
R_{i}^{-1}, M_{p}^{9} V R_{i}, M_{p}^{3} R_{i} R_{j}, M_{p}^{6} V R_{i}^{-1} R_{j}^{-1} .
$$

These are KK-particles, KK-monopoles, M2-branes and M5-branes respectively. Now let us turn on some flux $C_{m n p}(1 \leq m<n<p \leq 7)$ and let us assume, for simplicity, that $C_{m n p} \neq 0$ for only one set of indices $m, n, p$. Let us consider a BPS-state (that preserves some fraction of the SUSY) with integer charges $Q^{i}, \widetilde{Q}_{i}, Q_{i j}, \widetilde{Q}^{i j}$. From this vector one can construct an $8 \times 8$ complex anti-symmetric central charge matrix $Z_{a b}=-Z_{b a}(a, b=1 \ldots 8)$ that is linear in the $Q$ 's. The procedure is as follows [13]. Let us define the periodic variable:

$$
\phi^{m n p} \equiv C_{m n p} R_{m} R_{n} R_{p}, \quad \phi^{m n p} \sim \phi^{m n p}+1 .
$$

Next, one defines:[

$$
\begin{aligned}
q^{i} & \equiv Q^{i}+\phi^{i j p} Q_{j p}, \\
\widetilde{q}_{i} & \equiv \widetilde{Q}_{i}, \\
\widetilde{q}^{i j} & \equiv \widetilde{Q}^{i j}+\phi^{i j p} \widetilde{Q}_{p}, \\
q_{i j} & \equiv Q_{i j}-\epsilon_{i j k l m n p} \widetilde{Q}^{k l} \phi^{m n p} .
\end{aligned}
$$

\footnotetext{
${ }^{1}$ We wish to thank the anonymous referee for pointing out a typo in the first line. This corrects two formulas in section (『).
} 
Here we have used the assumption that $\phi_{m n p} \neq 0$ for only one set of indices $m, n, p$ (that we are going to take to be 1,6,7 later on). Otherwise, we will also have terms that are quadratic and cubic in $\phi$. One way to think about these equations is that the presence of the fractional $\phi_{m n p}$ creates effective fractional charges. For example, there is an effective fractional membrane charge if there is an M5-brane with a $C$-field turned on.

The central charge matrix is now given by:

$$
Z_{a b}=\sum_{1 \leq m<n \leq 7}\left(M_{p}^{3} R_{m} R_{n} q_{m n}+i M_{p}^{6} V R_{m}^{-1} R_{n}^{-1} \widetilde{q}^{m n}\right) \Gamma_{a b}^{m n}+\sum_{m=1}^{7}\left(M_{p}^{9} V R_{m} \widetilde{q}_{m}+i R_{m}^{-1} q^{m}\right) \Gamma_{a b}^{m 8} .
$$

Here we have used the anti-symmetric

$$
\Gamma_{a b}^{p q}=-\Gamma_{a b}^{q p}=-\Gamma_{b a}^{p q}, \quad p, q=1 \ldots 8, \quad a, b=1 \ldots 8
$$

which are the generators of $S O(8)$ in the spinor representation $\underline{\mathbf{8}}_{s}$. The BPS bound from the matrix $Z$ is that the mass squared of a state with given charge should be at least the maximal eigenvalue of $Z^{\dagger} Z$.

We shall now apply this formula to various BPS states in the theory.

\subsection{The 5-brane tension}

How much energy does it cost to separate the 5-brane from the Taub-NUT space?

We set $C_{167}$ to a nonzero value and set $\widetilde{Q}_{7}=1$ and $\widetilde{Q}^{67}=N$. We also set $\phi^{167}=$ $C_{167} R_{1} R_{6} R_{7}$. The central charge matrix is given by:

$$
Z=M_{p}^{9} V R_{7} \Gamma^{78}+i N M_{p}^{6} V R_{6}^{-1} R_{7}^{-1} \Gamma^{67}+i C_{167} M_{p}^{6} V R_{7} \Gamma^{16}
$$

The maximal eigenvalue of this matrix is:

$$
M_{p}^{6} V \sqrt{\left(M_{p}^{3} R_{7}+N R_{6}^{-1} R_{7}^{-1}\right)^{2}+C_{167}^{2} R_{7}^{2}}
$$

We compare this to the separate masses of the Taub-NUT alone and the M5-brane alone. The sum of the masses is:

$$
M_{p}^{6} V\left(\sqrt{M_{p}^{6} R_{7}^{2}+C_{167}^{2} R_{7}^{2}}+N R_{6}^{-1} R_{7}^{-1}\right)
$$


Thus, the bound state energy is:

$$
M=M_{p}^{6} V\left(\sqrt{M_{p}^{6} R_{7}^{2}+C_{167}^{2} R_{7}^{2}}+N R_{6}^{-1} R_{7}^{-1}-\sqrt{\left(M_{p}^{3} R_{7}+N R_{6}^{-1} R_{7}^{-1}\right)^{2}+C_{167}^{2} R_{7}^{2}}\right)
$$

Let us take the limit $M_{p}^{3} R_{6} R_{7}^{2} \rightarrow \infty$. We obtain $\left(C \equiv M_{p}^{-3} C_{167}\right)$ :

$$
M \approx N M_{p}^{6} V R_{6}^{-1} R_{7}^{-1}\left(1-\frac{1}{\sqrt{1+C^{2}}}\right) .
$$

Thus, the tension of each 5-brane effectively decreases by $\sqrt{1+C^{2}}$ when it is bound to the Taub-NUT.

\subsection{Momentum States}

The result above can be interpreted simply as a rescaling of the metric $g_{11}$ in the $1^{\text {st }}$ direction. To see this, let us compare the energy of a KK-particle in the $1^{\text {st }}$ direction to the energy of a KK-particle with momentum in the $2^{\text {nd }}$ direction.

For momentum in the $1^{\text {st }}$ direction we find:

$$
\begin{aligned}
Z & =M_{p}^{9} V R_{7} \Gamma^{78}+i N M_{p}^{6} V R_{6}^{-1} R_{7}^{-1} \Gamma^{67}+i C_{167} M_{p}^{6} V R_{7} \Gamma^{16}+i k R_{1}^{-1} \Gamma^{18} \\
& \equiv x \Gamma^{78}+i y \Gamma^{67}+i z \Gamma^{16}+i v \Gamma^{18}
\end{aligned}
$$

The mass is:

$$
\sqrt{(x+y+v)^{2}+z^{2}}-\sqrt{(x+y)^{2}+z^{2}} \longrightarrow \frac{x}{\sqrt{x^{2}+z^{2}}} v
$$

The last result is in the limit $x, z \gg y \gg v$. Thus the energy of a massless particle with $k$ units of momentum in the $1^{\text {st }}$ direction is:

$$
\frac{k}{\sqrt{1+C^{2}}} R_{1}^{-1}
$$

This suggests that we define:

$$
\widetilde{R}_{1} \equiv \sqrt{1+C^{2}} R_{1}
$$

The KK-mass is then $\widetilde{R}_{1}^{-1}$. For particles with momentum in the $2^{\text {nd }}$ direction we find:

$$
\begin{aligned}
Z & =M_{p}^{9} V R_{7} \Gamma^{78}+i N M_{p}^{6} V R_{6}^{-1} R_{7}^{-1} \Gamma^{67}+i C_{167} M_{p}^{6} V R_{7} \Gamma^{16}+i k R_{2}^{-1} \Gamma^{28} \\
& \equiv x \Gamma^{78}+i y \Gamma^{67}+i z \Gamma^{16}+i v \Gamma^{28}
\end{aligned}
$$

The mass is then just $k R_{2}^{-1}$. 


\subsection{Massive Particles}

As we have seen, the $N$ M5-branes are stuck at the center of the Taub-NUT space where their tension is minimal. Intuitively, this suggests that small fluctuations of the world-volume of the M5-branes are described by a massive field. A Taub-NUT space has a $U(1)$ isometry. When $C_{167}=0$, a fluctuation of the M5-brane in the Taub-NUT directions is charged under that $U(1)$ because at the center of the Taub-NUT geometry the $U(1)$ is embedded in the local $S O(4)$ isometry of the tangent space.

Thus, we should check what is the mass of a BPS state with $U(1)$ charge. We therefore set $Q^{7}=k$ and calculate:

$$
Z=\left(M_{p}^{9} V R_{7}+i k R_{7}^{-1}\right) \Gamma^{78}+i N M_{p}^{6} V R_{6}^{-1} R_{7}^{-1} \Gamma^{67}+i M_{p}^{6} C_{167} V R_{7} \Gamma^{16}
$$

Now the maximal eigenvalue is:

$$
M_{p}^{6} V \sqrt{\left(M_{p}^{3} R_{7}+N R_{6}^{-1} R_{7}^{-1}\right)^{2}+\left(C_{167} R_{7}+k M_{p}^{-6} V^{-1} R_{7}^{-1}\right)^{2}}
$$

The energy of the excitation is therefore:

$M_{p}^{6} V\left(\sqrt{\left(M_{p}^{3} R_{7}+N R_{6}^{-1} R_{7}^{-1}\right)^{2}+\left(C_{167} R_{7}+k M_{p}^{-6} V^{-1} R_{7}^{-1}\right)^{2}}-\sqrt{\left(M_{p}^{3} R_{7}+N R_{6}^{-1} R_{7}^{-1}\right)^{2}+C_{167}^{2} R_{7}^{2}}\right)$.

In the limit $M_{p}^{3} R_{6} R_{7}^{2} \rightarrow \infty$ this becomes:

$$
k \frac{C}{\sqrt{1+C^{2}}} R_{7}^{-1}
$$

\subsection{Tensor fluxes}

We can also calculate the energy of fluxes of the anti-self-dual 2-form field that is part of the low-energy tensor multiplet of an M5-brane. Because $C_{167}$ explicitly breaks Lorentz invariance, we should discuss fluxes in various directions separately. We set $Q_{i j}=N_{i j}$ for $N_{i j}$ units of tensor flux in the direction $i, j$. If $T_{i j k}$ is the anti-self-dual 3-form field-strength on the M5-brane then $N_{i j}=2 \pi T_{i j 0} R_{i} R_{j}$ for a single M5-brane.

Let us first set only $N_{23} \neq 0$. The central charge matrix is:

$$
\begin{aligned}
Z & =M_{p}^{9} V R_{7} \Gamma^{78}+i N M_{p}^{6} V R_{6}^{-1} R_{7}^{-1} \Gamma^{67}+i C_{167} M_{p}^{6} V R_{7} \Gamma^{16}+M_{p}^{3} N_{23} R_{2} R_{3} \Gamma^{23} \\
& \equiv x \Gamma^{78}+i y \Gamma^{67}+i z \Gamma^{16}+u \Gamma^{23}
\end{aligned}
$$


The BPS bound is:

$$
\sqrt{\left(x+\sqrt{u^{2}+y^{2}}\right)^{2}+z^{2}}
$$

In the limit $M_{p}^{3} R_{6} R_{7}^{2} \rightarrow \infty, x, z \gg y, u$ we obtain the energy of the flux:

$$
\begin{aligned}
E_{23} & =\sqrt{\left(x+\sqrt{u^{2}+y^{2}}\right)^{2}+z^{2}}-\sqrt{(x+y)^{2}+z^{2}}=\frac{\sqrt{u^{2}+y^{2}}-u}{\sqrt{x^{2}+z^{2}}} \\
& =\frac{\sqrt{1+M_{p}^{-6} N^{-2} N_{23}^{2} R_{1}^{-2} R_{4}^{-2} R_{5}^{-2}}-1}{\sqrt{1+C^{2}}} M_{p}^{6} N R_{1} R_{2} R_{3} R_{4} R_{5} \\
& \longrightarrow \frac{N_{23}^{2} R_{2} R_{3}}{2 \sqrt{1+C^{2}} N R_{1} R_{4} R_{5}}=\frac{N_{23}^{2} R_{2} R_{3}}{2 N \widetilde{R}_{1} R_{4} R_{5}} .
\end{aligned}
$$

The last line is in the limit $M_{p} R_{i} \gg 1$ for $i=1,4,5$. Next we check a flux with one index in the direction of $C_{167}$. Let us take $N_{12} \neq 0$. We find:

$$
\begin{gathered}
Z=M_{p}^{9} V R_{7} \Gamma^{78}+i N M_{p}^{6} V R_{6}^{-1} R_{7}^{-1} \Gamma^{67}+i C_{167} M_{p}^{6} V R_{7} \Gamma^{16}+M_{p}^{3} N_{12} R_{1} R_{2} \Gamma^{12} \\
\equiv x \Gamma^{78}+i y \Gamma^{67}+i z \Gamma^{16}+v \Gamma^{12} . \\
E_{12}=\sqrt{v^{2}+x^{2}+y^{2}+z^{2}+2 \sqrt{v^{2} x^{2}+x^{2} y^{2}+v^{2} z^{2}}}-\sqrt{(x+y)^{2}+z^{2}} \\
\longrightarrow \frac{\sqrt{v^{2}\left(1+C^{2}\right)+y^{2}}-y}{1+C^{2}} \longrightarrow \frac{v^{2} \sqrt{1+C^{2}}}{2 y} \\
=\frac{N_{12}^{2} \sqrt{1+C^{2}} R_{1} R_{2}}{2 N R_{3} R_{4} R_{5}}=\frac{N_{12}^{2} \widetilde{R}_{1} R_{2}}{2 N R_{3} R_{4} R_{5}} .
\end{gathered}
$$

We see that these results support the claim that we have to rescale the first coordinate by a factor of $\sqrt{1+C^{2}}$.

\subsection{Strings}

So far we described the excitations of a possibly free theory. Now we would like to take the number of M5-branes to be $N=2$ and separate the M5-branes along the $6^{\text {th }}$ direction. Let the separation be $\phi R_{6}$. We expect to find strings, made by M2-branes stretched between the M5-branes, with a tension proportional to $\phi$. We also have to establish the coefficient of the kinetic term, $(\partial \phi)^{2}$, in the low energy effective action. We can do that by taking $R_{2} \gg R_{6}$ and calculate the energy of and M5-brane that wraps the diagonal of the $2-6$ directions. 
This is described by $\phi\left(x_{2}\right)=l x_{2} R_{2}^{-1} R_{6}$. The corresponding central charge matrix is:

$$
\begin{aligned}
Z & =M_{p}^{9} V R_{7} \Gamma^{78}+i N M_{p}^{6} V R_{6}^{-1} R_{7}^{-1} \Gamma^{67}+i l M_{p}^{6} V R_{2}^{-1} R_{7}^{-1} \Gamma^{27}+i C_{167} M_{p}^{6} V R_{7} \Gamma^{16} \\
& \equiv x \Gamma^{78}+i y \Gamma^{67}+i z \Gamma^{16}+i w \Gamma^{27} .
\end{aligned}
$$

The energy is:

$$
E=\sqrt{x^{2}+y^{2}+z^{2}+w^{2}+2 \sqrt{x^{2} y^{2}+x^{2} w^{2}+w^{2} z^{2}}}-\sqrt{(x+y)^{2}+z^{2}} \approx \frac{\sqrt{x^{2}+z^{2}}}{2 x y} w^{2}
$$

This is:

$$
\sqrt{1+C^{2}} \frac{M_{p}^{6} V R_{6}}{2 N R_{7}} l^{2}=\frac{M_{p}^{6} \widetilde{R}_{1} R_{2} R_{3} R_{4} R_{5}}{2 N}\left(\frac{R_{6} l}{R_{2}}\right)^{2} .
$$

We compare this to:

$$
\frac{1}{2 N} \widetilde{R}_{1} \cdots R_{5}(\partial \phi)^{2}
$$

and find that:

$$
\varphi \equiv M_{p}^{3} \phi
$$

has the normalized kinetic energy.

To calculate the tension of the string we gradually change $\phi$ from 0 to $2 \pi$. When it is $2 \pi$ we calculate the mass of $k$ strings stretched on the $2^{\text {nd }}$ direction:

$$
\begin{aligned}
Z & =M_{p}^{9} V R_{7} \Gamma^{78}+i N M_{p}^{6} V R_{6}^{-1} R_{7}^{-1} \Gamma^{67}+k M_{p}^{3} R_{2} R_{6} \Gamma^{26}+i C_{167} M_{p}^{6} V R_{7} \Gamma^{16} \\
& \equiv x \Gamma^{78}+i y \Gamma^{67}+i z \Gamma^{16}+w \Gamma^{26}
\end{aligned}
$$

We find $E=w$. The energy of the string in the limit $R_{6} \rightarrow \infty$ is thus:

$$
M_{p}^{3} R_{2} \phi
$$

The tension of the string wrapped in a direction not including the $1^{\text {st }}$ is therefore $T \equiv \varphi$.

For the mass of strings stretched in the $1^{\text {st }}$ direction we calculate:

$$
\begin{aligned}
Z= & M_{p}^{9} V R_{7} \Gamma^{78}+i N M_{p}^{6} V R_{6}^{-1} R_{7}^{-1} \Gamma^{67}+k M_{p}^{3} R_{1} R_{6} \Gamma^{16}+i C_{167} M_{p}^{6} V R_{7} \Gamma^{16} \\
\equiv & x \Gamma^{78}+i y \Gamma^{67}+(w+i z) \Gamma^{16} \\
& E=\sqrt{(x+y+w)^{2}+z^{2}}-\sqrt{(x+y)^{2}+z^{2}} \approx \frac{x+y}{\sqrt{(x+y)^{2}+z^{2}}} w
\end{aligned}
$$


The energy of the string in the limit $R_{6} \rightarrow \infty$ is thus:

$$
\frac{1}{\sqrt{1+C^{2}}} M_{p}^{3} R_{1} \phi=\frac{1}{1+C^{2}} M_{p}^{3} \widetilde{R}_{1} \phi
$$

We note that in these calculations we assume that the metric on the $\varphi$-moduli space is constant. In $5+1 \mathrm{D}$, supersymmetry implies that the metric on the tensor-multiplet moduli space is flat. However, in our case we can only use the $S O(4,1)$ subgroup of the $S O(5,1)$ Lorentz group. So, in principle there can be a nontrivial metric on the moduli space [15].

\subsection{Summary}

Based on the BPS analysis we found the following facts.

- We have to rescale the metric in the $1^{\text {st }}$ direction so that $\widetilde{R}_{1}=\sqrt{1+C^{2}} R_{1}$ where $C=M_{p}^{-3} C_{167}$. This way the energy of massless particles with momentum $p$ is given by the Lorentz invariant expression $|p|$.

- The tension of each M5-brane is smaller by a factor of $\sqrt{1+C^{2}}$ when it is at the center of the Taub-NUT (relative to infinity).

- There appears to be a massive particle in the spectrum with mass

$$
m_{0} \equiv \frac{C}{\sqrt{1+C^{2}}} R_{7}^{-1}
$$

- The energy of tensor fluxes is as it should be if expressed in terms of $\widetilde{R}_{1}$. Tensor fluxes in direction 1,2 have energy:

$$
E_{12}=\frac{N_{12}^{2} \widetilde{R}_{1} R_{2}}{2 N R_{3} R_{4} R_{5}},
$$

while tensor fluxes in direction 2,3 have energy:

$$
E_{23}=\frac{N_{23}^{2} R_{2} R_{3}}{2 N \widetilde{R}_{1} R_{4} R_{5}} .
$$

- When the M5-branes are separated there appear to be strings in the spectrum. The tension of the strings is proportional to the separation. The tension seems to be smaller by a factor of $\left(1+C^{2}\right)$ for strings stretched in the $1^{\text {st }}$ direction relative to strings stretched in the directions $2 \ldots 5$. 


\section{Gravity solutions}

We have seen in section (3) that a Taub-NUT space with nonzero boundary conditions for the 3-form field along the circle at infinity creates a potential that pins M5-branes to the origin. We have also seen that some excitations of the M5-brane become massive. In this section we will explain the mechanism that is responsible for these effects. For that purpose we will describe the classical supergravity solution that corresponds to this Taub-NUT space. The solution is a good approximation when the curvature and field strength are small and that is true for $M_{p} R_{7} \gg 1$ and $M_{p}^{-3} C_{167} \ll 1$. We will also describe the solution of the Taub-NUT space with $N$ M5-branes at the center. This solution is a good approximation either when $N$ is large or $N=0$.

\subsection{The solution}

We start with a configuration of $Q_{5}$ D5-branes and $Q_{3}$ D3-branes in type-IIB theory oriented along $\left(x_{0}, x_{2}, x_{3}, x_{4}, x_{5}, x_{6}\right)$ and $\left(x_{0}, x_{2}, x_{3}, x_{7}\right)$ respectively. We have kept $x_{1}$ to be the $11^{\text {th }}$ direction so as to remain consistent with the notations of the previous sections.

Under an S-duality this will become a system of $Q_{5}$ NS5-branes and $Q_{3}$ D3-branes. We define the following terms:

$$
H_{3}=1+Q_{3} / r, \quad H_{5}=1+Q_{5} / r
$$

and $r=\sqrt{\left(x^{8}\right)^{2}+\left(x^{9}\right)^{2}+\left(x^{10}\right)^{2}}$. $Q_{i}$ depends on the number $N_{i}$ of D-branes and also on $M_{p}$. We will use the explicit form of the $Q_{i}$ later. The metric for the NS5-D3 configuration is (see 16, 17, 18]):

$$
d s^{2}=H_{3}^{-1 / 2} d s_{023}^{2}+H_{3}^{1 / 2} d s_{456}^{2}+H_{5} H_{3}^{-1 / 2} d s_{7}^{2}+H_{5} H_{3}^{1 / 2} d s_{89,10}^{2}
$$

After a series of T-dualities in the $4^{\text {th }}$ and $5^{\text {th }}$ directions, we get a configuration of $Q_{5}$ NS5branes and $Q_{3}$ D5 oriented along $\left(x_{0}, x_{2}, x_{3}, x_{4}, x_{5}, x_{6}\right)$ and $\left(x_{0}, x_{2}, x_{3}, x_{4}, x_{5}, x_{7}\right)$ respectively with metric:

$$
d s^{2}=H_{3}^{-1 / 2} d s_{02345}^{2}+H_{3}^{1 / 2} d s_{6}^{2}+H_{5} H_{3}^{-1 / 2} d s_{7}^{2}+H_{5} H_{3}^{1 / 2} d s_{89,10}^{2}
$$


Basically this is our starting configuration. The whole chain of dualities was done only to calculate the metric for this configuration. The directions $x^{6}, x^{7}$ are on a square torus. To go to an inclined torus we make the following transformations:

$$
\begin{aligned}
& x^{6}=y^{6} \sec \theta+y^{7} \sin \theta, \\
& x^{7}=y^{7} \cos \theta .
\end{aligned}
$$

$y^{i}$ are the new coordinates. Observe that $x^{j}=y^{j}$ for $j \neq 6,7$. Therefore only the 6,7 part of the metric will undergo some change, and it will look like:

$$
\begin{aligned}
d s^{2} & =H_{3}^{1 / 2}\left(d y^{6} \sec \theta+d y^{7} \sin \theta\right)^{2}+H_{5} H_{3}^{-1 / 2}\left(d y^{7} \cos \theta\right)^{2} \\
& =H_{3}^{1 / 2}\left(d y^{6} \sec \theta\right)^{2}+\left(H_{3}^{1 / 2} \sin ^{2} \theta+H_{5} H_{3}^{-1 / 2} \cos ^{2} \theta\right)\left(d y^{7}\right)^{2}+2 H_{3}^{1 / 2} \tan \theta d y^{6} d y^{7}
\end{aligned}
$$

The rest of the components of the metric will remain the same. Now under a T-duality along $y^{7}$ we get a $\left(Q_{5}\right.$-centered $)$ Taub-NUT space and $Q_{3}$ D4-branes. The Taub-NUT space has a nontrivial metric along $\left(y_{7}, y_{8}, y_{9}, y_{10}\right)$ and the D4-branes are oriented along $\left(y_{2}, y_{3}, y_{4}, y_{5}\right)$.

The metric for this configuration is:

$$
d s^{2}=H_{3}^{-1 / 2} d s_{02345}^{2}+h H_{5}\left(d y^{6}\right)^{2}+h\left(d y^{7}+B_{7 i} d y^{i}\right)^{2}+H_{5} H_{3}^{1 / 2} d s_{89,10}^{2}
$$

where $h^{-1}=H_{3}^{1 / 2} \sin ^{2} \theta+H_{5} H_{3}^{-1 / 2} \cos ^{2} \theta$ and $i=8,9,10$. Observe that the KK gauge field $A_{i}=B_{7 i}$ and $B_{7 i}$ comes from the NS5 brane. The Taub-NUT circle is along $y_{7}$.

There is also an antisymmetric two form background coming from the inclination of the torus. It is given by:

$$
B^{(N S)}=h H_{3}^{1 / 2} \tan \theta d y^{6} \wedge\left(d y^{7}+B_{7 i} d y^{i}\right)
$$

The coefficient goes to a constant $T=\tan \theta$ at infinity. The dilaton behaves as:

$$
e^{2 \phi}=h H_{5} / H_{3}
$$

The string coupling constant, $g$, has been set to one.

We now lift this configuration to M-theory. The M-theory direction is $x^{1}$. The various components of the metric are ( $a, b$ are 10-dimensional indices):

$$
G_{a b}=\left(h H_{5} / H_{3}\right)^{-1 / 3} g_{a b}, \quad G_{a 1}=0, \quad G_{11}=\left(h H_{5} / H_{3}\right)^{2 / 3}
$$


and the three form background is:

$$
C_{3}=M_{p}^{3} h H_{3}^{1 / 2} \tan \theta d x_{1} \wedge d y^{6} \wedge\left(d y^{7}+B_{7 i} d y^{i}\right)
$$

At infinity, the value of the 3 -form flux becomes $M_{p}^{3} C \equiv M_{p}^{3} \tan \theta$.

We can still subtract from $C_{3}$ a constant because this does not affect the field-strength. We do it so as to fix the boundary condition $C_{3}=0$ at $r=0$. This is because the radius of the circle in the $7^{\text {th }}$ direction shrinks to zero and and if $C_{3}(0) \neq 0$ we would get a singularity in the field-strength at the origin. We calculate the constant piece to be:

$$
\frac{M_{p}^{3} Q_{3} \tan \theta}{Q_{3} \sin ^{2} \theta+Q_{5} \cos ^{2} \theta} \text {. }
$$

Now let us study the background geometry alone, setting the number $Q_{3}$ of M5-branes, to zero. We obtain the metric:

$$
\begin{aligned}
d s^{2}= & \left(h H_{5} / H_{3}\right)^{2 / 3} d x_{1}^{2}+\left(h H_{5}\right)^{-1 / 3} H_{3}^{-1 / 6}\left(d x_{0}^{2}+d x_{2}^{2}+d x_{3}^{2}+d x_{4}^{2}+d x_{5}^{2}\right)+\left(h H_{5}\right)^{2 / 3} H_{3}^{1 / 3}\left(d y^{6}\right)^{2} \\
& +h^{2 / 3}\left(H_{5} / H_{3}\right)^{-1 / 3}\left(d x_{7}+B_{7 i} d x_{i}\right)^{2}+h^{-1 / 3} H_{5}^{2 / 3} H_{3}^{5 / 6}\left(d x_{8}^{2}+d x_{9}^{2}+d x_{10}^{2}\right) \\
= & \left(h H_{5}\right)^{2 / 3} d x_{1}^{2}+\left(h H_{5}\right)^{-1 / 3}\left(d x_{0}^{2}+d x_{2}^{2}+d x_{3}^{2}+d x_{4}^{2}+d x_{5}^{2}\right) \\
& +\left(h H_{5}\right)^{2 / 3}\left(d y^{6}\right)^{2}+h^{2 / 3} H_{5}^{-1 / 3}\left(d x_{7}+B_{7 i} d x_{i}\right)^{2}+h^{-1 / 3} H_{5}^{2 / 3}\left(d x_{8}^{2}+d x_{9}^{2}+d x_{10}^{2}\right)
\end{aligned}
$$

where:

$$
H_{5}=1+\frac{R}{r}, \quad h^{-1}=\sin ^{2} \theta+H_{5} \cos ^{2} \theta=1+\frac{R \cos ^{2} \theta}{r} .
$$

We also find:

$$
\begin{aligned}
M_{p}^{-3} C_{3} & =h\left(H_{3}\right)^{1 / 2} \tan \theta d x_{1} \wedge d y^{6} \wedge\left(d x_{7}+B_{7 i} d x_{i}\right) \\
& =\left(1+\frac{R \cos ^{2} \theta}{r}\right)^{-1} \tan \theta d x_{1} \wedge d y^{6} \wedge\left(d x_{7}+B_{7 i} d x_{i}\right)
\end{aligned}
$$

as $r \rightarrow \infty$ we see that

$$
M_{p}^{-3} C_{3}(\infty)=\tan \theta d x_{1} \wedge d y^{6} \wedge\left(d x_{7}+B_{7 i} d x_{i}\right) \equiv C d x_{1} \wedge d y^{6} \wedge\left(d x_{7}+B_{7 i} d x_{i}\right)
$$

\subsection{Small $r$}

For $r \rightarrow 0$ we can still trust our supergravity solution as long as the background value of the three form potential is sufficiently small. In this limit $h^{-1}=H_{5} /\left(1+C^{2}\right)$ in the absence of 
M5 brane. $H_{5}$, on the other hand, goes as $R r^{-1}$ for small $r$. The metric as seen by the M5 brane is nonsingular and behaves as follows:

$$
\begin{aligned}
d s^{2} & =\left(1+C^{2}\right) d x_{1}^{2}+d x_{02345}^{2}+\left(1+C^{2}\right) d x_{6}^{2} \\
& +\left(1+C^{2}\right) \frac{r}{R}\left(d y_{7}+A_{i} d y_{i}\right)^{2}+\frac{R}{r}\left(d r^{2}+r^{2} d \Omega_{8910}^{2}\right)
\end{aligned}
$$

We have scaled the coordinates by $\left(1+C^{2}\right)^{1 / 6}$ to get the above metric. Here $y_{7}$ and the angular variables $\Omega_{8,9,10}$ can be taken to parameterize an $\mathbf{S}^{3}$. If we change variables to $r=u^{2}$ we see that the $r, x_{7}, \Omega_{8,9,10}$ parameterize a smooth 4-dimensional point at $r=0$, as in the ordinary Taub-NUT space. As $r \rightarrow 0$, the field $C_{3}$ behaves as:

$$
C_{3} \rightarrow C\left(1+C^{2}\right) M_{p}^{3}(r / R) d x_{1} \wedge d y^{6} \wedge\left(d x_{7}+B_{7 i} d x_{i}\right)
$$

It is easy to check that the field strength, $F_{4}=d C_{3}$, has a finite magnitude as $r \rightarrow 0$.

\subsection{The pinning potential}

To calculate the potential we have to compute $\sqrt{\operatorname{det} G}$ along the M5-brane directions. This is given by the following expression:

$$
\operatorname{det} G=G_{00} G_{11} \cdots G_{55}=H_{3}^{-3 / 2} H_{5}^{-1}\left(H_{3}^{1 / 2} \sin ^{2} \theta+H_{5} H_{3}^{-1 / 2} \cos ^{2} \theta\right)
$$

Now to calculate the potential as seen by the M5 brane we put $H_{3}=1$. This reduces to

$$
\sqrt{\operatorname{det} G}=\left(H_{5}^{-1} \sin ^{2} \theta+\cos ^{2} \theta\right)^{1 / 2}
$$

As discussed earlier one can trust supergravity solution if $C \ll 1$. Therefore we have the following limits:

- For $r \rightarrow \infty$ all the harmonic functions become 1 and so $\sqrt{\operatorname{det} G}=1$.

- Near $r \rightarrow 0, H_{5} \rightarrow R / r$ and we can neglect $\sin ^{2} \theta$. This gives

$$
\sqrt{\operatorname{det} G}=\frac{1}{\sqrt{1+C^{2}}}
$$

This is precisely the reduction expected from the BPS analysis in the previous sections. 


\subsection{Lorentz invariance}

The origin $r=0$ is a smooth point for the metric (4). If we rescale:

$$
\widetilde{x}_{1} \equiv \sqrt{1+C^{2}} x_{1}
$$

then at the vicinity of $r=0$ the $S O(5,1)$ Lorentz invariance is restored. This is the same rescaling found in section (3). As we will see in section (5), the low-energy theory that describes the M5-branes breaks $S O(5,1)$ Lorentz invariance, but at lowest order the breaking term involves only the fermions.

Let us also mention that even though the metric is smooth, the 4-form field strength, $d C_{3}$, is discontinuous at $r=0$. It is nevertheless finite and that will play an important role in generating the Lorentz-breaking fermion term (see section (6)).

\subsection{Small fluctuations}

We can expand the potential (6) in small $r$. To leading order,

$$
\sqrt{\operatorname{det} G}=\frac{1}{\sqrt{1+C^{2}}}+\frac{C^{2}}{2\left(1+C^{2}\right)^{1 / 2}} \frac{r}{R}+O(r)^{2} .
$$

If we change coordinates to $u=r^{1 / 2}$ and rescale $x_{1}$ by the factor $\sqrt{1+C^{2}}$, we see that fluctuations of the position of the M5-brane have an effective potential of

$$
\frac{C^{2}}{2\left(1+C^{2}\right)} M_{p} u^{2} \text {. }
$$

We conclude that the fluctuations become massive with the same mass as predicted by the BPS calculation (2).

\section{Decoupling limits}

We now wish to study limits of the previous constructions where gravity can be decoupled. To be concrete, we will concentrate on the example of $N$ M5-branes at the center of a Taub-NUT space with $C_{167}$-flux turned on.

There are two kinds of decoupling arguments that we can utilize. The first is a low-energy argument where we set to infinity the scale of all the excitations that we wish to discard. 
Thus, we say that $\mathcal{N}=4 \mathrm{SYM}$ theory describes $N$ coincident D3-branes when we set the string-scale to infinity, thereby decoupling the massive string states [1]. We will call this type of argument "kinematical". Sometimes we are forced to keep the scale of excitations, that we wish to decouple, finite. Another type of decoupling argument is possible if we can set the coupling constant between those excitations and the excitations of our theory to zero. This type of argument was introduced in [8] for the decoupling of bulk string states from the little-string theory. The bulk states have masses of order the string scale $M_{s}$ which is the same scale as that of the little-string theory. The decoupling is argued to occur in the limit of zero string coupling constant. We will call this a "dynamical" argument.

\subsection{Kinematical decoupling}

We have seen in section (3) that an M5-brane at the center of a Taub-NUT space with the field $C_{167}$ turned on has massive BPS states. Let us make a list of the various energy scales that we found in section (3). In the limit of $C \rightarrow 0$, we have:

- $M_{p}$ is the Planck scale.

- $R_{7}^{-1}$ is the scale of KK-excitations far away from the center.

- $M_{p} C^{1 / 3}$ is the energy-scale of the binding energy per unit volume of the 5 -brane.

- $C R_{7}^{-1}$ is the energy scale of excitations of the 5-brane.

In a low-energy decoupling limit we must set the first three scales to infinity. The Planck scale, $M_{p}$ must be set to infinity in order to decouple gravity. The scale $M_{p} C^{1 / 3}$ must be set to infinity so that we will not have to consider local fluctuations where a small portion of the M5-brane escapes to infinity. Finally, the scale $R_{7}^{-1}$ will be sent to infinity so as to keep the scale of Kaluza-Klein particles that are far from the center small. We will keep $C R_{7}^{-1}$ finite. Finally, we should also take $M_{p} R_{7} \rightarrow \infty$. The reason is as follows. If we reduce from M-theory to type-IIA along $R_{7}$, the Taub-NUT space becomes a D6-brane and the M5-branes becomes NS5-branes. The string scale is $M_{s}^{2}=M_{p}^{3} R_{7}$ and this must be set to infinity.t Moreover, we want the scale that is set by the tension of the D6-brane to be much higher than the scale set by the tension of the M5-branes, otherwise we could not decouple

\footnotetext{
${ }^{2}$ We thank S. Sethi for pointing this out.
} 
the $6+1 \mathrm{D} U(1)$ gauge field on the D6-brane. The implies that the string coupling constant should be large, and hence $M_{p} R_{7} \rightarrow \infty$.

The decoupled theory that we obtain seems to be a new type of $5+1 \mathrm{D}$ theory that has not been encountered before.

\subsection{Dynamical decoupling}

In this limit we take $M_{p} \rightarrow \infty$ but we wish to keep $C$ finite. Since we wish to keep $C R_{7}^{-1}$ finite as well, we are forced to keep the scale $R_{7}^{-1}$ itself finite. This means that we must find another argument for the decoupling of Kaluza-Klein excitations that are far from the center. Such an argument has to be dynamical, namely that the coupling constant between these states and the states of the $5+1 \mathrm{D}$ theory is proportional to inverse powers of $M_{p}$. This argument is similar in spirit to the argument made in [8] for the decoupling of bulk string states from the little-string theory. The bulk states have masses of order the string scale $M_{s}$ which is the same scale as that of the little-string theory. The decoupling is argued to occur in the limit of zero string coupling constant.

In our case, in the limit of keeping both $R_{7}$ and $C$ finite, we saw in section (3) that the metric in the $1^{\text {st }}$ direction has to be rescaled by a factor of $\sqrt{1+C^{2}}$ in order to preserve Lorentz invariance, at least to leading order. By this we mean that the energy of massless particles with low momentum $p$, will be $E=|p|$ no matter what the direction of $p$ is. We have also seen that with this rescaling the energy of tensor fluxes in directions 2,3 (i.e. not including the direction of the $C$-flux) is:

$$
E_{23}=\frac{N_{23}^{2} R_{2} R_{3}}{2 N \widetilde{R}_{1} R_{4} R_{5}},
$$

where $N_{23}$ is an integer. Fluxes in directions 1,2 have energy:

$$
E_{12}=\frac{N_{12}^{2} \widetilde{R}_{1} R_{2}}{2 N R_{3} R_{4} R_{5}}
$$

which also respects the invariance under interchange of directions 1 and 2 .

At higher orders in the momentum expansion, we expect Lorentz invariance to be broken. As we shall see in section (6), it is the fermions that first exhibit the breakdown of Lorentz invariance. 


\section{Low-energy description}

What is the low-energy description of these 5+1D theories? A regular M5-brane is described, at low-energies, by a tensor multiplet of $\mathcal{N}=(2,0)$ supersymmetry. It contains an anti-selfdual tensor field and 5 scalars. In our case, we have argued that the M5-brane becomes pinned in 4 out of the 5 transverse directions. Thus, it is reasonable to assume that 4 scalars become massive and the low-energy description is an anti-self-dual tensor field and a single scalar. Together with fermions, that would make up a single tensor multiplet of $\mathcal{N}=(1,0)$ supersymmetry and the equations of motion would be Lorentz invariant at the lowest order in the derivative expansion.

\subsection{The fermions: a puzzle}

What about the fermions? At first sight, there seems to be a puzzle. We have seen that 4 scalars become massive. In terms of $\mathcal{N}=(1,0)$ the 4 scalars are part of a hyper-multiplet. However, a hypermultiplet in $5+1 \mathrm{D}$ contains chiral fermions and these cannot be made massive. Furthermore, imagine that we turn on $C$ gradually from 0 to its present value. The theory at $C=0$ has a (massless) hypermultiplet that contributes to the gravitational anomaly. How did this part of the anomaly disappear at $C \neq 0$ ?

The resolution of both puzzles is that the theory at $C \neq 0$ is not Lorentz invariant. Thus, we should only consider the $S O(4,1) \subset S O(5,1)$ subgroup of Lorentz-invariance and therefore only $4+1 \mathrm{D}$ supersymmetry. In $4+1 \mathrm{D}$, a hypermultiplet can be given a mass.

Let $\lambda$ be the fermion that lives on an M5-brane. It is in the representation $(\underline{4}, \underline{4})$ of $\operatorname{Spin}(4,1) \times \operatorname{Spin}(5)$ where $\operatorname{Spin}(5)$ is the rotation of the transverse directions.

$\operatorname{Spin}(4,1)$ invariance in directions $0,2 \ldots 5$ suggests that the effective coupling would be:

$$
\sim \bar{\lambda} \Gamma^{1} \Omega \lambda
$$

Here $\Gamma^{1}$ is one of the $5+1 \mathrm{D}$ Dirac matrices and $\Omega$ acts on the $\operatorname{Spin}(5)$ R-symmetry indices. Furthermore, $\operatorname{Spin}(3)$ invariance in directions 8,9,10 suggests that $\Omega$ should be a constant $\operatorname{Spin}(3) \subset \operatorname{Spin}(5)$ invariant matrix. After dimensional reduction to $4+1 \mathrm{D}$ the term must reduce to an ordinary mass term for the fermions of the hypermultiplet because the theory 
would be Lorentz invariant. This means that $\Gamma^{1} \Omega$ must be the identity on the fermions of the hypermultiplet. Starting with a vector multiplet with 16 supersymmetries in $4+1 \mathrm{D}$ we can consider an $\mathcal{N}=1$ subgroup (8 supersymmetries) of the SUSY algebra. The fermions that go into the hypermultiplet can be separated from those that go into the vector multiplet (in $4+1 \mathrm{D})$ by their transformation under the Spin(4) that, in the original setting, corresponds to rotations in directions $7,8,9,10$. The fermions of the hypermultiplet are invariant and this determines $\Omega=\Gamma^{6}$ - the 11D Dirac matrix in the $6^{\text {th }}$ direction. Note that we cannot set $\Omega$ to the identity because then the effective action will be just like a coupling to a constant gauge field that can be gauged away.

\subsection{The supergravity mechanism}

What is the explicit mechanism by which our fermions get a mass? If one places an M5brane in a region where the 4-form field strength, $F$, of M-theory is nonzero but still small, the fermions, $\lambda$, on the world-volume of an M5-brane (that are part of the tensor multiplet) couple to it schematically as $\lambda \lambda F$. One way to see this is by expanding the action with the fermionic zero-modes of the M5-brane solution. Recall that M-theory has a term in the effective action that is of the form [23] $\bar{\psi}^{M} \Gamma^{P Q} \psi^{N} F_{M P Q N}$, where $M, P, Q, N=0 \ldots 10$, $\psi^{M}$ is the gravitino field, $F_{M P Q N}$ is the 4 -form field-strength and $\Gamma^{P Q}$ is an anti-symmetric product of two Dirac matrices. After reduction to the zero-modes on the M5-brane solution we can find the effective coupling between the 5+1D fermions and the external 4 -form fieldstrength. (Note that the M5-brane solution has strong curvature but we can still use it for this discussion since supersymmetry determines that term uniquely.)

In our case, let $\mu=0 \ldots 5$ a direction parallel to the M5-brane. Let $A=6 \ldots 10$ be a direction orthogonal to the M5-brane. We are interested in the coupling between the components $F_{\mu A B C}$ of the field-strength and the fermions $\lambda$ from the $(2,0)$ tensor-multiplet.

This coupling has to be of the form $F_{\mu A B C} \bar{\lambda} \Gamma^{\mu} \Gamma^{A B C} \lambda$, where $\Gamma^{A B C}$ acts on the $S p(2) \mathrm{R}$ symmetry indices only and $\Gamma^{\mu}$ acts on the space-time $\operatorname{Spin}(5,1)$ spinor indices only.

We have seen in section (4.2) that the 4 -form field-strength, $F_{4}$, approaches a constant magnitude as $r \rightarrow 0$ but it is still discontinous because its direction depends on the path along the transverse directions $(7,8,9,10)$ in which we take the limit $r \rightarrow 0$. At first sight 
this would seem to suggest that the $F \lambda \lambda$ term has a scalar-field dependent coefficient. We believe, however, that the correct procedure is to expand the gravitino fields in the presence of the M5-brane as $\psi^{M} \sim \lambda \psi_{0}^{M}$, where $\psi_{0}^{M}$ are the gravitino zero-modes and the $\lambda$ 's depend only on directions $0 \ldots 5$ (along the M5-brane). Then, we have to plug this back into the M-theory coupling, $\bar{\psi}^{M} \Gamma^{P Q} \psi^{N} F_{M P Q N}$, with $F_{M N P Q}$ taken from (5). We believe that this will produce the term suggested in (7).

We could be a bit more precise here. Observe that the value of $F_{r 167}$ near $r \rightarrow 0$ is a constant given by $C\left(1+C^{2}\right) R^{-1}$. Therefore we expect the fermions to pick up a mass proportional to the value of $F$ at the origin. However this is not the case. It is of course true that the $S O(4,1)$-invariant mass involves $F$ but there is also a contribution from the zero modes of the gravitino in the picture. In the presence of the background $C$ field, the normalisable zero modes also pick up contributions from the $C$ field in such a way that the zero modes are actually suppressed by inverse powers of $\left(1+C^{2}\right)$. To see this, consider the D5-NS5 brane configuration. Let us assume that the system supports a normalisable gravitino zero mode $\psi_{0}^{7}(r)$. We now go to the slanted torus by the transformation given in section (4.1) and then make a T-duality along $x_{7}$. Using the T-duality rules the combined effect now gives a zero mode suppressed by $\left(1+C^{2}\right)^{-1 / 2}$. Now integrating out the zero modes using the 11-dimensional term (with $x_{1}$ being scaled by a factor of $\sqrt{1+C^{2}}$ ) we see that after dimensional reduction to $4+1 \mathrm{D}$ the fermions get the same mass as the bosons.

\section{Various BPS states in $6+1 \mathrm{D}$}

We will now drop the M5-branes from the story and concentrate only on the Taub-NUT space with the C-flux. For the purposes of the discussion it is convenient to think of it as a $6+1 \mathrm{D}$ theory, although we do not necessarily claim that it is decoupled from gravity. We will return to that question in section (7.1).

We can calculate the tension of various BPS states of this $6+1 \mathrm{D}$ "theory". We can do this using the same techniques as described in section (3). We can even check some of the statements for small $C$ using the solution in section (4.1). As in section (3) we will assume that the theory is compactified on $\mathbf{T}^{6}$ with radii $R_{1}, \ldots, R_{6}$. We will express the results in 
terms of the rescaled radii:

$$
\widetilde{R}_{1} \equiv \sqrt{1+C^{2}} R_{1}, \quad \widetilde{R}_{6} \equiv \sqrt{1+C^{2}} R_{6}
$$

We have calculated the energy of the following BPS objects:

- Kaluza-Klein particles: They have energy:

$$
\sqrt{\frac{k_{1}^{2}}{\widetilde{R}_{1}^{2}}+\frac{k_{2}^{2}}{R_{2}^{2}}+\cdots+\frac{k_{5}^{2}}{R_{5}^{2}}+\frac{k_{6}^{2}}{\widetilde{R}_{6}^{2}}}
$$

- M2-branes: M2-branes that are stretched in directions $I \neq J$ have the following masses, according to the dimension of the intersection of the plane of the membrane with the plane of the C-flux.

- For $I, J=2,3,4,5$ we have the mass:

$$
\frac{1}{\sqrt{1+C^{2}}} M_{p}^{3} R_{I} R_{J}
$$

- For $I, J$ with $I=1,6$ and $J=2,3,4,5$ we find the mass:

$$
\frac{1}{\sqrt{1+C^{2}}} M_{p}^{3} \widetilde{R}_{I} R_{J}
$$

- The BPS formula for M2-branes in direction 1, 6 gives:

$$
\frac{1}{\sqrt{1+C^{2}}} M_{p}^{3} \widetilde{R}_{1} \widetilde{R}_{6}
$$

which again agrees with the supergravity calculation as in (4.1), for small $C$.

- M5-branes: This again depends on whether the M5-brane hyper-plane contains both the $1^{\text {st }}$ and $6^{\text {th }}$ directions or just one of them.

- For M5-branes in direction 1 . .5 we find the mass:

$$
\frac{1}{1+C^{2}} M_{p}^{6} \widetilde{R}_{1} R_{2} R_{3} R_{4} R_{5}
$$

- For M5-branes in direction 1,3 . .6 we find the mass:

$$
\frac{1}{1+C^{2}} M_{p}^{6} \widetilde{R}_{1} R_{3} R_{4} R_{5} \widetilde{R}_{6}
$$

- Electric fluxes: For this purpose we can think of the Taub-NUT as a D6-brane (after reduction on the $7^{\text {th }}$ direction). 
- For electric flux in the $1^{\text {st }}$ direction we find the central charge:

$$
Z=M_{p}^{9} V R_{7} \Gamma^{78}-i C_{167} R_{1} R_{7} \Gamma^{68}+M_{p}^{3} R_{1} R_{7} \Gamma^{17}+i C_{167} M_{p}^{6} V R_{7} \Gamma^{16}
$$

and the energy is:

$$
\frac{\sqrt{1+C^{2}} \widetilde{R}_{1}}{2 M_{p}^{3} R_{2} R_{3} R_{4} R_{5} \widetilde{R}_{6}} .
$$

- For flux in the $2^{\text {nd }}$ direction we find:

$$
Z=M_{p}^{9} V R_{7} \Gamma^{78}+M_{p}^{3} R_{2} R_{7} \Gamma^{27}+i C_{167} M_{p}^{6} V R_{7} \Gamma^{16}
$$

The energy is:

$$
\frac{\sqrt{1+C^{2}} R_{2}}{2 M_{p}^{3} \widetilde{R}_{1} R_{3} R_{4} R_{5} \widetilde{R}_{6}} .
$$

- Magnetic fluxes: We take the magnetic flux to be in direction $I, J$. We will distinguish three cases:

- For $I, J=2, \ldots 5$, for example $I, J=2,3$, we find that the central charge is:

$$
Z=M_{p}^{9} V R_{7} \Gamma^{78}+i M_{p}^{3} R_{1} R_{4} R_{5} R_{6} R_{7} \Gamma^{23}-C_{167} R_{1} R_{4} R_{5} R_{6} R_{7} \Gamma^{45}+i C_{167} M_{p}^{6} V R_{7} \Gamma^{16},
$$

and the energy is:

$$
\frac{M_{p}^{3} \widetilde{R}_{1} R_{4} R_{5} \widetilde{R}_{6}}{2 \sqrt{1+C^{2}} R_{2} R_{3}} .
$$

- For $I=2, \ldots 5$ and $J=1,6$, for example, $I=2$ and $J=1$, we find:

$$
Z=M_{p}^{9} V R_{7} \Gamma^{78}+i M_{p}^{3} R_{3} R_{4} R_{5} R_{6} R_{7} \Gamma^{12}+i C_{167} M_{p}^{6} V R_{7} \Gamma^{16}
$$

and the energy is:

$$
\frac{M_{p}^{3} R_{3} R_{4} R_{5} \widetilde{R}_{6}}{2 \sqrt{1+C^{2}} \widetilde{R}_{1} R_{2}}
$$

- For $I, J=1,6$, we find the energy:

$$
\frac{C}{\sqrt{1+C^{2}}} M_{p}^{6} R_{2} R_{3} R_{4} R_{5} R_{7} .
$$




\subsection{The large $C$ limit}

Perhaps the most interesting limit to study is that of $C \rightarrow \infty$. [5 Specifically, let us consider the following limit:

$$
M_{p} \rightarrow \infty, C \rightarrow \infty, M_{p}^{3} C^{-1} \rightarrow \text { fixed. }
$$

This limit has been studied in [20]. They argued that a D6-brane in the limit of noncommutative geometry [10]- [12] is described by a decoupled 6+1D theory. Keeping $M_{p}^{3} C^{-1}$ finite makes sures that the effective Yang-Mills coupling constant $g_{Y M}^{2}=M_{p}^{-3} C$ is fixed. The proposal of a decoupled theory is also related to a previous suggestion of [19] that a non-commutative version of the $6+1 \mathrm{D}$ theory that should have been the M(atrix)-model of M-theory on $T^{6}$ could perhaps avert the problems explained in [22, 21] and actually be a decoupled theory. We will not address the issue of decoupling in this paper. (Following a correspondence with O. Aharony, we tend to believe that the $6+1 \mathrm{D}$ theory is decoupled, as suggested in [20], but has a continuum of states like a 10+1D theory.)

Nevertheless, we will point out that all the energies of the BPS states studied in the previous section, except the magnetic flux in directions 1,6 , have a finite limit if $M_{p}^{3} C^{-1}$ is kept fixed and $R_{7}$ is also kept finite.

The mass of the M2-branes is proportional to $1 / g_{Y M}^{2}$, the mass of the M5-branes is proportional to $1 / g_{Y M}^{4}$ and the energy of electric-fluxes in directions orthogonal to 1 and 6 is proportional to $g_{Y M}^{2}$. It is interesting to note that the M2-branes and M5-branes are becoming light when $g_{Y M}^{2}$ is large (compared to the radii $\widetilde{R}_{1}, R_{2}, \ldots, R_{5}, \widetilde{R}_{6}$ ).

\section{Discussion}

We have seen that the dynamics near the origin of a Taub-NUT space with a $C$-field turned on at $\infty$ can be used to construct various decoupled theories. We have suggested two types of theories. These theories can be obtained by placing M5-branes as probes and taking either a low-energy limit or a less understood dynamical decoupling limit. In all these examples, the dynamics depends only on the type of singularity at $r=0$. The virtue of using a Taub-NUT space is that we can easily use BPS arguments as in section (3).

\footnotetext{
${ }^{3}$ We wish to thank S. Sethi for pointing this limit out.
} 
We can repeat the discussions of the previous sections with D-branes of various dimensions instead of M5-branes or with M2-branes. When we discuss D-branes we can turn on various RR-fields or NSNS 2-form fields at infinity. These fields can have various numbers of indices along the direction of the branes. Thus, we will obtain theories that are either Lorentz invariant or have a Lorentz-breaking term that is characterized by a vector or a tensor.

Alternatively, we can compactify the $5+1 \mathrm{D}$ theory that we found on the M5-branes on $\mathbf{T}^{d}$ and look for a low-energy description of the resulting $(6-d)$-dimensional theory. Since the Lorentz-breaking direction (the $1^{\text {st }}$ direction in the notation of section (3)) can be either compactified or not, we obtain low-energy descriptions that are either Lorentz-invariant or have a vector Lorentz-breaking term.

The two questions, one regarding replacing M5-branes with D-branes or M2-branes and the other regarding the low-energy description of compactified theories, are overlapping. For example, if we compactify the theory of ordinary M5-branes, i.e. the (2,0)-theory, on $\mathbf{T}^{2}$ we obtain the theory of D3-branes, i.e. $U(N) \mathcal{N}=4 \mathrm{SYM}$ at low-energy. If we further compactify the theory of D3-branes on $\mathbf{S}^{1}$ we obtain the theory of D2-branes that at the IR limit flows to the $\operatorname{Spin}(8)$ theory of M2-branes at a certain point in moduli space [24-26].

These theories seem to provide a mechanism for fixing the position of branes in an ambient space. It would be interesting to generalize these constructions to cases, with probably less supersymmetry, in which more than 4 coordinates of the position of a D-brane are fixed. This might have applications for brane-world scenarios as in [27. 31.

Let us also mention that our models are related to the models studied in [32]. Both models are U-dual to the elliptic brane configurations of 33 .

Another question that we hope to address in a later paper is the large $N$ limit of this theory, along the lines of the AdS/CFT correspondence 34, 35, 36.

It would also be interesting to study the limit of large $C$ as discussed in (7.1).

\section{Acknowledgments}

It is a pleasure to thank M. Berkooz, M.B. Green, A. Kapustin, S. Mukhi, A. Sen and S. Sethi for helpful discussions. We are also grateful to O. Aharony and T. Banks for helpful 
correspondence. G.R. gratefully acknowledges the hospitality of the Mathematical Institute at the University of Oxford, where part of this work was done.

The research of K.D. is supported by Department of Energy grant No. DE-FG0290ER4054442. The research of O.J.G was supported by National Science Foundation grant No. PHY98-02484. The research of G.R. is supported by NSF grant number NSF DMS9627351. 


\section{References}

[1] E. Witten, "Bound States of Strings and p-Branes," Nucl. Phys. B460 (1996) 335-350, hep-th/9510135

[2] A. Strominger, "Open p-Branes," Phys. Lett. B383 (1996) 44-47, hep-th/9512059

[3] Michael R. Douglas and G. Moore, "D-branes, Quivers, and ALE Instantons," hepth/9603167

[4] T. Banks, M.R. Douglas and N. Seiberg, "Probing F-theory With Branes," Phys. Lett. B387 (1996) 278-281, hep-th/9605199

[5] N. Seiberg, "IR Dynamics on Branes and Space-Time Geometry," Phys. Lett. B384 (1996) 81-85, hep-th/9606017

[6] J.D. Blum and K. Intriligator, "New Phases of String Theory and 6d RG Fixed Points via Branes at Orbifold Singularities," Nucl. Phys. B506 (1997) 199,hep-th/9705044

[7] I.R. Klebanov and E. Witten, "Superconformal Field Theory on Three Branes at a Calabi- Yau Singularity," Nucl. Phys. B536 (1998) 199-218,hep-th/9807080

[8] N. Seiberg, "New Theories in Six-Dimensions and Matrix Description of M-theory on $T^{5}$ and $T^{5} / Z_{2}$," hep-th/9705221, Phys. Lett. B408 (1997) 98

[9] A. Connes, "Noncommutative Geometry", Academic Press1994

[10] A. Connes, M.R. Douglas and A. Schwarz, "Noncommutative Geometry and Matrix Theory: Compactification on Tori," JHEP. 02 (1998) 003, hep-th/9711162

[11] M.R. Douglas and C. Hull, "D-branes and the Noncommutative Torus," JHEP. 02 (1998) 008, hep-th/9711165

[12] N. Seiberg and E. Witten, "String Theory and Non-Commutative Geometry," hepth/9908142

[13] E. Witten, "String Theory Dynamics in Various Dimensions," Nucl. Phys. B443 (95) 85, hep-th/9503124

[14] N.A. Obers and B. Pioline, "U-duality and M-theory," hep-th/9809039

[15] N. Seiberg, "Five Dimensional SUSY Field Theories, Non-trivial Fixed Points and String Dynamics," hep-th/9608111 
[16] A. Tseytlin, "Harmonic Superpositions of M-branes", Nucl. Phys. B475 (1996) 149, hep-th/9604035

[17] A. Tseytlin, "Composite BPS configurations of p-branes in 10 and 11 dimensions," Class. Quant. Grav. 14 (1997) 2085-2105, hep-th/9702163

[18] J. P. Gauntlett, "Intersecting Branes", hep-th/9702163

[19] O. Aharony, T. Banks and E. Siverstein, unpublished.

[20] M. Alishahiha, Y. Oz and M.M. Sheikh-Jabbari, "Supergravity and Large N Noncommutative Field Theories," JHEP. 9911 (1999) 007, hep-th/9909215

[21] A. Sen, "D0 Branes on $T^{n}$ and Matrix Theory," hep-th/9709220

[22] N. Seiberg, "Why is the Matrix Model Correct?," Phys. Rev. Lett. 79 (1997) 3577-3580, hep-th/9710009

[23] E. Cremmer and B. Julia, "The SO(8) Supergravity," Nucl. Phys. B159 (1979) 141

[24] S. Sethi and L. Susskind, "Rotational Invariance in the M(atrix) Formulation of Type IIB Theory," Phys. Lett. B400 (1997) 265-268, hep-th/9702101

[25] T. Banks and N. Seiberg, "Strings from Matrices," Nucl. Phys. B497 (1997) 41-55, hep-th/9702187

[26] N. Seiberg, "Notes on Theories with 16 Supercharges," hep-th/9705117

[27] N. Arkani-Hamed, S. Dimopoulos and G. Dvali, "Phenomenology, Astrophysics and Cosmology of Theories with Sub-Millimeter Dimensions and TeV Scale Quantum Gravity," Phys. Rev. D59 (1999) 86004, hep-ph9807344

[28] I. Antoniadis, N. Arkani-Hamed, S. Dimopoulos and G. Dvali, "New Dimensions at a Millimeter and Superstrings at a TeV," Phys. Lett. B436 (1998) 257, hep-ph/9804398

[29] N. Arkani-Hamed, S. Dimopoulos and G. Dvali, "The Hierarchy Problem and New Dimensions at a Millimeter," Phys. Lett. B429 (1998) 263, hep-ph/9803315

[30] Z. Kakushadze and H. Tye, "Brane World," Nucl. Phys. B548 (1999) 180, hepth/9809147

[31] L. Randall and R. Sundrum, "An alternative to compactification," Phys. Rev. Lett. 83 (1999) 4690, hep-th/9906064 
[32] C.V. Johnson, A. Peet and J. Polchinski, "Gauge Theories and Excision of Repulson singularities," hep-th/9911161

[33] E. Witten, "Solutions Of Four-Dimensional Field Theories Via M Theory," Nucl. Phys. B500 (1997) 3-42,hep-th/9703166

[34] J.M. Maldacena, "The Large $N$ Limit of Superconformal Field Theories and Supergravity," hep-th/9711200

[35] A. Hashimoto and N. Itzhaki, "Non-Commutative Yang-Mills and the AdS/CFT Correspondence," Phys. Lett. B465 (1999) 142, hep-th/9907166

[36] J.M. Maldacena and J.G. Russo, "Large N Limit of Non-Commutative Gauge Theories," JHEP. 9909 (1999) 025, hep-th/9908134 